\title{
Notice of erratum
}

\author{
Mitsukuni Suenaga - Nobuyuki Mizunuma - Daigo Shouji - Eiji Shinozaki · \\ Satoshi Matsusaka - Keisho Chin · Masatoshi Oya - Toshiharu Yamaguchi · \\ Tetsuichiro Muto $\cdot$ Kiyohiko Hatake
}

Published online: 29 May 2009

(C) Springer 2009

\section{Erratum to: J Gastroenterol (2008) 43:842-848 \\ DOI 10.1007/s00535-008-2242-1}

M. Suenaga, et al. Modified irinotecan plus bolus 5-fluorouracil/L-leucovorin for metastatic colorectal cancer at a single institution in Japan. J Gastroenterol (2008) 43:842848.

Please note that there are errors in the above article:

On page 842 (Abstract) on lines 17-20 in the left column, "6.1", "17.4", "28.8" and "8.9" should be "8.6", "27.8", "30.9" and " 14.5 ", respectively.

On page 844 , beginning on line 1 in the right column, "The median PFS of the 47 patients was 6.1 months (95\% CI, 6.0-9.9 months)" should be "The median PFS of the 47 patients was 8.6 months."

On page 844 , beginning on line 8 in the right column, "The median OS of the 47 patients was 17.4 months (95\%
CI, 15.9-22.9 months)" should be "The median OS of the 47 patients was 27.8 months."

On page 844 on line 11 in the right column, " 28.8 " and "8.9" should be "30.9" and "14.5", respectively.

On page 846 on lines 38-39 in the left column, "6.1", "2.6" and "17.4" should be "8.6", "13" and " $27.8 "$ ", respectively.

On page 846 on line 46 in the left column, "28.8" should be "30.9".

The online version of the original article can be found under doi:10.1007/s00535-008-2242-1.

M. Suenaga · N. Mizunuma - D. Shouji · E. Shinozaki ·

S. Matsusaka $\cdot$ K. Chin $\cdot$ K. Hatake $(\square)$

Department of Medical Oncology, Cancer Institute Hospital,

Japanese Foundation for Cancer Research,

3-10-6 Ariake, Koto-ku, Tokyo 135-8550, Japan

e-mail: khatake@jfcr.or.jp

M. Oya - T. Yamaguchi - T. Muto

Division of Gastroenterological Surgery,

Cancer Institute Hospital,

Japanese Foundation for Cancer Research,

Tokyo, Japan 